\title{
Take a deep breath
}

\section{Want to know what's going on inside your lungs? Conventional imaging techniques are not much use, but by inhaling a magnetized gas you could get a clear picture of your airways. Erica Klarreich investigates.}

J ames Brookeman wasn't surprised that the student volunteers taking part in his medical experiments enjoyed themselves. All they had to do was inhale xenon gas, the side effects of which are a strangely low voice and a feeling of euphoria. "They giggled quite a while after inhaling," says Brookeman, a biomedical engineer at the University of Virginia in Charlottesville. "They loved it."

While the volunteers enjoyed themselves, they were helping to change the face of medical imaging. By tweaking the magnetic properties of xenon and helium, Brookeman and others are improving magnetic resonance imaging (MRI) so that it can reveal parts of the body previously invisible to the technique.

By offering a fresh window into the lungs, these researchers should improve our understanding of diseases such as asthma and emphysema. "This is the technique of the future for looking at lung function," says Edwin van Beek, who is working on the technique at the University of Sheffield, UK. And in the long term, the experiments could lead to cheaper MRI devices and new ways of studying brain disease.

\section{All in a row}

Conventional MRI makes use of the fact that hydrogen nuclei act like tiny magnets. When a patient lies inside an MRI machine, some hydrogen nuclei in water atoms in their body's tissues become polarized they align themselves with the powerful magnetic field the machine produces. The device then delivers pulses of radio waves that temporarily tilt the nuclei, inducing an electrical current in a coil of wire in the machine. Because the nuclei in different tissues fall back into line at different rates, MRI machines can distinguish between these tissues to produce images of the brain, for example, or the kidneys.

But not all organs can be imaged in this way. The lungs contain little water, and so have been off-limits to MRI scans. Other imaging techniques, such as positron-emission tomography (PET), which tracks the movement of radionuclides around the body, can image the lungs. But PET scans expose the patient to radiation, making them impractical tools for studying diseases, such as asthma, which would require patients to have regular scans.

Such problems led Brookeman and others

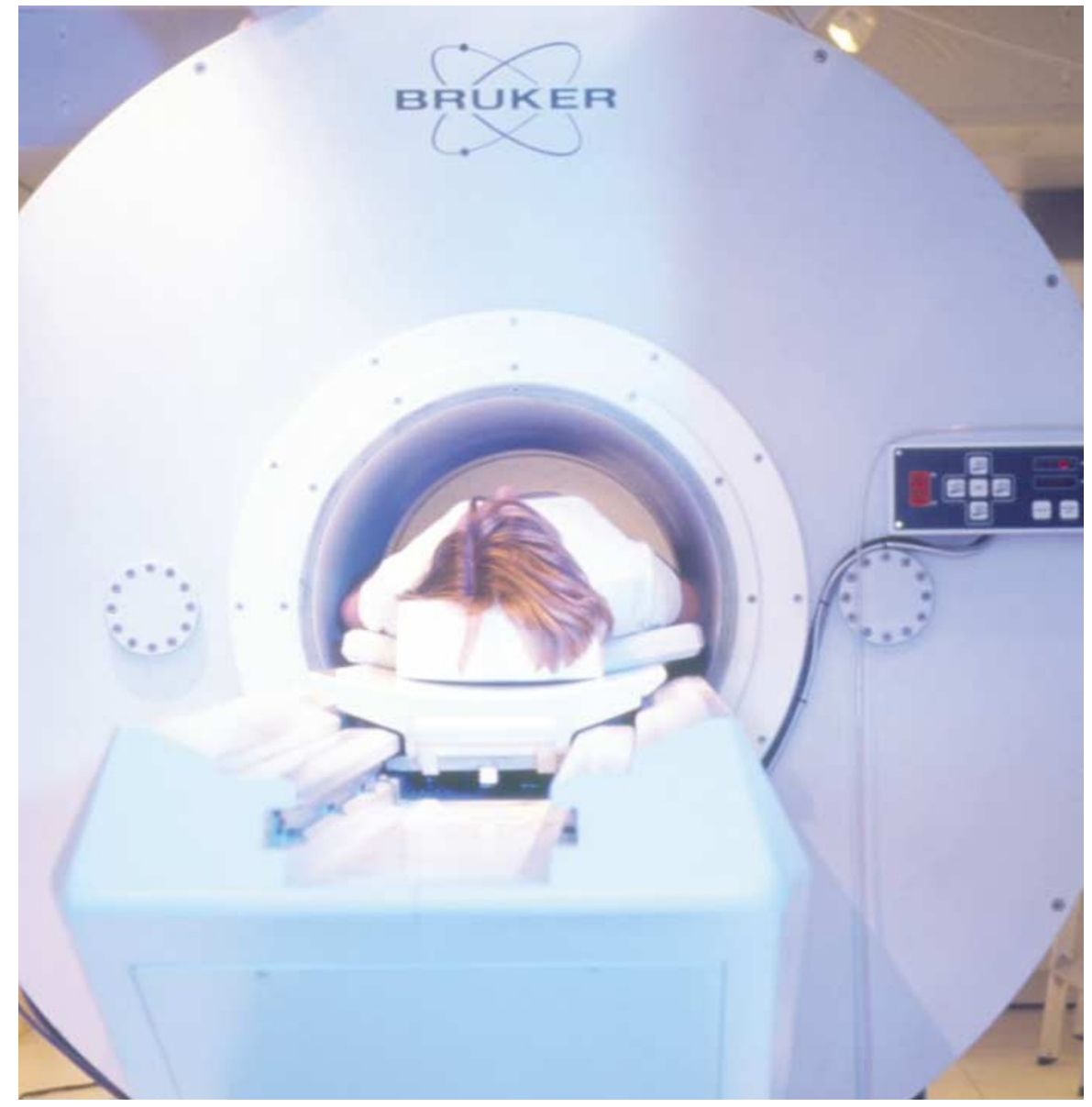

Magnetic resonance imaging offers an insight into the body, but until now it could not show the lungs.
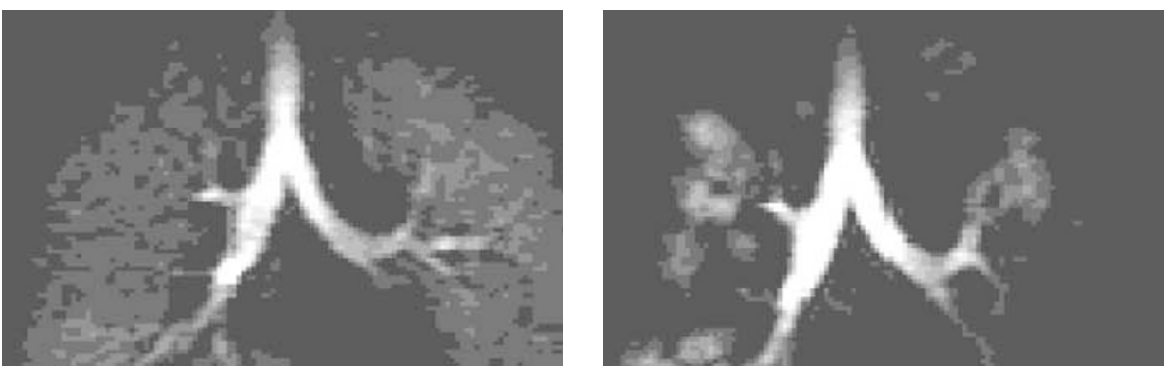

Clear view: normal lungs (left) and a simulated asthma attack taken using gas-based imaging.

to explore an alternative technique: filling the airways of the lungs with a gas that can respond to MRI. The idea dates back to the early 1990s, and is the brainchild of William Happer and Gordon Cates, then both at Princeton University in New Jersey. The two physicists were studying the process of hyperpolarization, which involves using a polarized beam of laser light to align the nuclei of gaseous atoms. Their work wasn't aimed at medicine - hyperpolarized helium-3 gas is used as a target in particle-scattering experiments - but they realized that a hyperpolarized gas should react to radio pulses in a similar way to aligned hydrogen nuclei.

In 1994, Cates and others described the first image taken using hyperpolarized xenon-129 gas in a lung, in this case from a 
dead mouse (M. S. Albert et al. Nature 370, 199-201; 1994). The resulting lung image was a spectacular success, and was soon followed by in vivo images of human and mouse lungs (see, for example, M. Ebert et al. Lancet 347, 1297-1299; 1996).

In theory, the technique offers significant benefits. A much greater proportion of nuclei are aligned in hyperpolarized gases than in water exposed to the magnetic fields in MRI, so the signals produced using the gas are about 100 times stronger. As a result, a hyperpolarized gas can image an entire lung in the few seconds it takes a patient to inhale, hold their breath, and exhale - a marked improvement over the hour or so it typically takes to get an image using conventional MRI. By taking images as a patient breathes in and out, the new technique can also produce dynamic images of airflow in and out of the lungs.

\section{Polar vision}

The treatment and diagnosis of asthma could be one area to benefit. In an asthma attack, branches of the tree-like structure of the lungs' airways either narrow or shut down. This June, Brookeman and his team described how they used hyperpolarized helium to detect which portions of the lungs were cut off during an asthma attack - areas where gas flow was reduced or eliminated showed up darker in the images (S. Samee et al. J. Allergy Clin. Immunol. 111, 1205-1211; 2003). "Parts of the lung totally closed; other parts remained normal," says Brookeman. "Before, it wasn't clear whether the narrowing happened everywhere or not."

Hyperpolarized gas MRI should also be valuable for testing the efficacy of asthma therapies, says Mitchell Albert, a radiologist who works on hyperpolarization at Brigham and Women's Hospital in Boston. "We'll be able to figure out if one treatment is better than another, what is the optimal time for medication, how frequently patients should use their medication, and so on," he says.

Another disease that may reap the benefits offered by hyperpolarized gas MRI is emphysema. This condition affects the walls of the small air sacs, called alveoli, that act as the interface between the lungs and the bloodstream. The walls break down, merging alveoli together to form much larger air sacs. This greatly reduces the surface area available for oxygen transport into the blood, leaving the sufferer fighting for breath.

Dmitriy Yablonskiy, a physicist at Washington University in St Louis, Missouri, is part of a team investigating the rate at which hyperpolarized gas diffuses through the lungs of emphysema patients. In a healthy lung, diffusion in the alveoli is relatively slow, as gas atoms can't move far before bumping into the edge of a sac. Diffusion in emphysema patients is faster, because the large alveoli

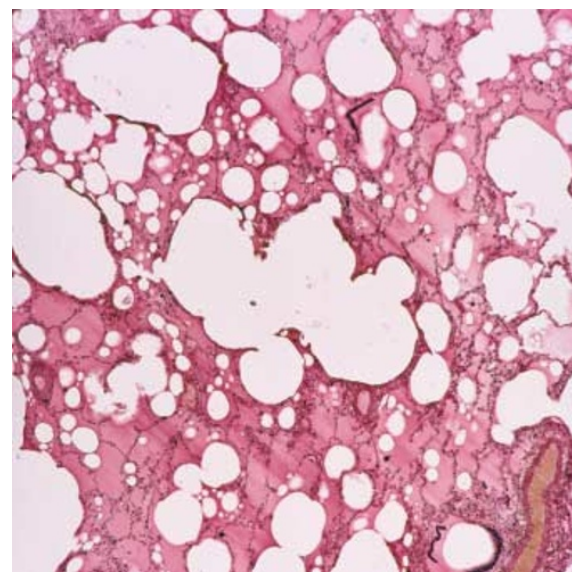

Gas-based imaging may allow rapid diagnosis of emphysema (below), in which the lungs' small airsacs become enlarged (above).

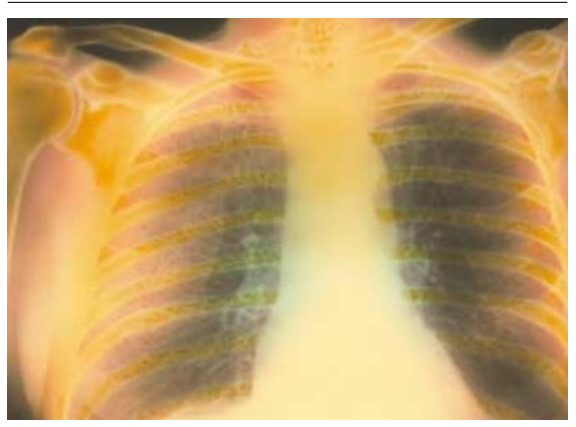

give the gas more room to move. "The diffusion coefficient is four times larger in emphysema patients than healthy people," says Yablonskiy.

Such discoveries could help to improve diagnosis. Brookeman's team is using diffusion measurements to study smokinginduced emphysema. He notes that patients do not usually start showing symptoms of the disease until after 20 pack-years of smoking (a pack-year is equivalent to smoking a packet of 20 cigarettes a day for a year). "But with a diffusion map of the lungs, it looks as though we can spot the effects of smoking after five pack-years," he says. "We can tell people that they should quit because their lungs are starting to deteriorate, even though they have no symptoms."

\section{Shadowy figures}

Armed with hyperpolarized gas and oxygen, researchers are optimistic that they will also be able to image blood clots in the lungs. Oxygen increases the speed at which hyperpolarized helium and xenon lose their polarization. Blood clots prevent oxygen from being absorbed in the lungs, so if a patient inhales oxygen, it will linger in the vicinity of the clot. If the patient then inhales hyperpolarized gas, areas where the oxygen hasn't been absorbed will give a much weaker MRI signal than other areas, showing up as a dark area on the scan.

But the lungs may be just the beginning xenon-129 has an added advantage that should allow it to image other organs. Unlike helium, xenon dissolves in the blood, reaching the brain and other organs within seconds of being inhaled. Albert is involved in a project to investigate whether xenon could be used to study brain plaques, lumps of tissue that build up in the nervous system and are associated with diseases such as multiple sclerosis. Albert hopes that xenon imaging will provide a way to distinguish between plaques caused by multiple sclerosis and those caused by other factors such as blood-flow problems.

Taking images with hyperpolarized gas MRI could be relatively cheap, says John Owers-Bradley, a physicist at the University of Nottingham, UK. Conventional MRI scanners use superconducting magnets to align hydrogen nuclei, and these need to be cooled by liquid helium. But in the new technique, the gas is already polarized, so a small magnetic field is needed to generate the final image. "We've produced good pictures with fields just one-tenth the usual strength," says Owers-Bradley. And compared with hydrogen atoms, which move out of alignment within seconds of a magnetic field being removed, isolated samples of hyperpolarized helium and xenon can remain polarized for more than a day. This means that the gas for a lung MRI can be prepared in advance, even in a separate facility, making it easier for small hospitals to adopt the technology.

\section{The $\mathbf{X}$ factor}

So far, most human trials of the technique have used helium-3 rather than xenon-129, mainly because helium is easier to hyperpolarize than xenon. But techniques for improving xenon polarization are currently being developed. This is important, as helium-3 does not occur in large quantities naturally. In fact, the only abundant source of the gas is nuclear-weapons programmes - helium-3 is a decay product of tritium, which is used in the trigger of hydrogen bombs. "In the early days, we'd usually have to deal with some Russian general who wanted the money put in a Swiss bank account," says Brookeman.

The US and Russian governments treat the gas as a strategic material, so although several companies are now buying helium-3 from governments and selling it to MRI researchers, no one is sure just how large the stocks are worldwide. The supply is probably enough for about 10 years' worth of imaging, Happer guesses. Xenon-129, by contrast, is relatively abundant: it makes up nearly onequarter of the xenon in the atmosphere.

With cheap and high-resolution images on the horizon, hyperpolarization looks set to boost medical imaging, transforming the way in which we study certain diseases. "I'm convinced it's going to be of great benefit," says Brookeman. His giggling students, it would seem, have spent their time wisely.

Erica Klarreich is a freelance writer in Berkeley, California. 